\title{
Rationale and design of a pragmatic clinical trial to assess the impact of self-monitoring blood pressure at home and self-titration of antihypertensive medication in poorly controlled hypertension: the ADAMPA study protocol
}

José Sanfélix-Genovés 1,2,3,4*, Clara L. Rodríguez-Bernal2,3, Irene Marco-Moreno ${ }^{4}$, Patricia Martinez-Ibañez ${ }^{4}$ Lucía Martinez-Ibañez ${ }^{4}$, María Bóveda-García ${ }^{4}$, Ignacio Barreira-Franch" ${ }^{4}$, Mercedes Calleja-Del Ser ${ }^{4}$, Greta Borrás-Moreno ${ }^{4}$, Eugenia Avelino-Hidalgo ${ }^{4}$, Marina Escrig-Veses ${ }^{4}$, Margherita Lauriano ${ }^{4}$, Margarita Giménez-Loreiro ${ }^{4}$, Laura Bellot-Pujalte ${ }^{4}$, Aníbal García-Sempere ${ }^{2,3}$, Salvador Peiró ${ }^{2,3}$, Gabriel Sanfélix-Gimeno ${ }^{2,3}$ and ADAMPA group

\begin{abstract}
Background: Lack of control of hypertension is one of the most prevalent problems encountered by general practitioners (GPS). Self-measured blood pressure monitoring at home (SMBP) and self-titration of medication could be a good strategy to improve hypertension management, however, evidence is limited and not conclusive. We aimed to assess the effectiveness, in the primary care setting, of an intervention that includes educational components, SMBP and self-titration of antihypertensive medication to decrease systolic blood pressure compared to usual care, in a population with poorly controlled hypertension, during a 12-month period.
\end{abstract}

Methods: Pragmatic, controlled, randomized, unblinded clinical trial with two parallel groups assigned in a ratio of 1:1 to self-management (which includes educational components, SBMP and self-titration of antihypertensive medication based on a patient's GP's pre-established adjustment plan) or to usual care (with educational components too).

Discussion: If the data from this trial show positive results, the study may contribute to a change of strategy in the treatment of hypertension, focusing on the patient as the main actor to achieve blood pressure control. Furthermore, this approach might contribute to the financial sustainability of the National Health Service.

Trial registration: This trial has been registered in the database with reference number EudraCT: 2016-003986-25. Registered 05 May 2017, https://www.clinicaltrialsregister.eu/ctr-search/search?query=2016-003986-25

Keywords: Self-monitoring, Blood pressure, Hypertension, Self-titration, Primary care, Pragmatic clinical trial

\footnotetext{
* Correspondence: sanfelix_jos@gva.es

${ }^{1}$ Centro de Salud de Nazaret, Departamento de Salud de Valencia Clínic-La Malvarrosa, Valencia, Spain

${ }^{2}$ Health Services Research Unit, FISABIO, Valencia, Spain

Full list of author information is available at the end of the article
}

(c) The Author(s). 2018 Open Access This article is distributed under the terms of the Creative Commons Attribution 4.0 International License (http://creativecommons.org/licenses/by/4.0/), which permits unrestricted use, distribution, and reproduction in any medium, provided you give appropriate credit to the original author(s) and the source, provide a link to the Creative Commons license, and indicate if changes were made. The Creative Commons Public Domain Dedication waiver (http://creativecommons.org/publicdomain/zero/1.0/) applies to the data made available in this article, unless otherwise stated. 


\section{Background}

The presence of hypertension is one of the most important issues in the global burden of disease [1]. In developed countries, the degree of control of hypertension has increased progressively over the last 15 years and has contributed to a decline in cardiovascular morbidity and mortality [2-8]. However, a recent study carried out in 12 European countries showed that more than $50 \%$ of patients treated for hypertension continued to have uncontrolled blood pressure (BP) [9] and that results are far from ideal. As a large part of hypertension management is carried out in primary care (PC) and it is one of the most prevalent problems encountered by General Practitioners (GP), interventions aimed at improving its management should preferably be made in this setting. Recent hypertension clinical guidelines put emphasis on self-measured blood pressure monitoring (SMBP) by patients and on team-based systems to manage the condition [10].

Self-measured blood pressure monitoring at home (SMBP) is practiced extensively nowadays. In the United Kingdom and Canada it is highly recommended by GPs and used by more than $30 \%$ of patients $[11,12]$. Systematic reviews have shown disparate information regarding the effectiveness of SMBP alone in reducing blood pressure (BP). On the other hand, self-monitoring in conjunction with co-interventions (including systematic medication titration by doctors, pharmacists, or patients; education; or lifestyle counseling) has been shown to lead to clinically significant BP reduction, which persists for at least 12 months. Nevertheless, the effectiveness of SMBP requires additional evaluation given that its definition in those studies is highly heterogeneous (different clinical protocols, different strategies for additional support and management) and given the fact that most studies have short follow-ups (1 year or less) [13, 14].

Regarding home titration of antihypertensive medication, evidence is more limited and shows mixed results. Two clinical essays, the TASMINH2 [15] and the TASMINH-SR [16], both in the United Kingdom and developed in the primary care setting by the same research team, are some of the most recent and interesting clinical trials carried out in this field. In these studies, SMBP together with self-titrate medications (according to a previously agreed plan), combined with telemedicine components, was compared with usual care. In both studies systolic blood pressure (SBP) decreased from baseline to 12 months, with significant differences between the intervention and control group (5.4 and $9.2 \mathrm{mmHg}$, respectively). Frequency of side effects was similar in both groups $[15,16]$. The TASMINH-SR study is of special relevance because it was carried out with high risk patients (with a personal history of stroke, ischemic cardiopathy, diabetes or kidney failure), a population of special interest to achieve BP targets [16]. On the other hand, a clinical trial carried out in the US in a low-income, predominantly minority population, aimed to determine whether health coaching, SMBP and home titration of antihypertensive medications could improve BP control compared with SMBP and health coaching alone. The results showed that both the home-titration arm and the no-home-titration arm had a reduction in SBP, with no significant differences between them from baseline to 6 months [17].

Finally, when interpreting hypertension studies over time, it is important to procede with caution, as the definition of the condition changes almost with every update of guidance. For instance, earlier versions of guidelines such as those of the the Joint National Committee (JNC) and of the European Society of Hypertension (ESH)/European Society of Cardiology (ESC), suggested more restrictive BP control objectives than recent versions (especially in patients over 60 years old, diabetics and patients with renal failure) [18-20]. These objectives may be modified again in the light of the results of recent studies [21-23].

\section{Study aim}

The primary aim of the ADAMPA TRIAL is to assess the effectiveness, in the primary care setting, of an intervention that includes educational components, SMBP and self-titration of antihypertensive medication to decrease SBP compared to usual care, in a population with poorly controlled hypertension, during a 12-month period. In addition, an extension with passive follow-up is planned for 24 months, collecting a reduced set of outcome variables as secondary variables.

\section{Main research questions}

1. Does a self-management intervention based on SMBP and self-titration medication allow for better control of blood pressure?

2. What is the impact of this intervention on adherence to treatments, lifestyle changes, quality of life, clinical outcomes and use of health services?

3. Is this intervention cost-effective?

4. What are patients', caregivers' and health professionals' views and experiences of SMBP plus self-titration in poorly controlled hypertension?

\section{Methods}

\section{Study design and setting}

The ADAMPA study is a pragmatic, controlled, randomized, unblinded clinical trial with two parallel groups assigned in a ratio of 1:1 to self-management (which includes educational components, SBMP and self-titration of antihypertensive medication based on a patient's GP's pre-established adjustment plan) or to usual care (with educational components too). 
All participants belong to a Health Area in the Valencia Region (Spain), with a population coverage of 345,500 inhabitants and a geographical area covering the north-east of the city of Valencia and some surrounding towns that are served by sixteen Primary Care Centers (PCC), two Hospitals and a Medical Specialty Centre.

This trial has been registered in the https://eudract.ema.europa.eu/ database with reference number EudraCT: 2016-003986-25.

\section{Study participants \\ Identification and recruitment}

Potential patients eligible to participate in the study will be selected by their General Practitioners (GP) among all patients attending their general practice (case-finding). In their general practice at the PCC, the GPs will inform patients of the study objective and in the case that they meet the inclusion criteria and none of exclusion criteria, GPs will give them the information sheet and informed consent form, responding to all queries and concerns.

\section{Eligibility criteria}

Eligibility criteria will aim to recruit patients with treated hypertension managed in primary care, who are aged 40 years or older, have a diagnosis of hypertension in their electronic history (coded), have a mean $\mathrm{BP}$ reading on the reference arm of SBP $>145$ or diastolic blood pressure (DBP) $>90 \mathrm{mmHg}$ on the baseline examination, who voluntarily accept participation in the study and sign the corresponding informed consent. The mean BP will be obtained as follows: In the first visit, BP should be measured on both arms. If there are differences, the reference arm should be that with the highest value of BP. Subsequently, the average $\mathrm{BP}$ of at least two measurements, in the sitting position, spaced 1-2 min apart should be calculated. If the first two readings are very different, an additional measurement should be done and the mean BP will be the average of the two readings considered valid [8].

\section{Exclusion criteria}

Exclusion criteria will include inability to self-manage their BP, including dementia or significant cognitive impairment (at the discretion of the researcher performing the recruitment), a history of orthostatic hypotension (fall $>20 \mathrm{mmHg}$ from SBP after taking the orthostatic position), $\mathrm{SBP}>200$ or $\mathrm{DBP}>100 \mathrm{mmHg}$ in the baseline examination, being prescribed more than 4 antihypertensive drugs, inclusion in another hypertension study or clinical trial, presence of tremor or neurological disease that makes it difficult to perform SMBP, presence of arrhythmia, presence of terminal illness, chronic incapacitation to leave home, an acute cardiovascular event in the last 3 months, hypertension managed directly by other specialist doctors outside the primary care environment, pregnant women or those actively seeking to become pregnant, having a household member already enrolled in the study and non- or temporary residents.

\section{Randomization}

Patients with uncontrolled hypertension will be randomized in a 1:1 ratio to receive either usual care or self-management using a computer randomization system via internet. Minimization will be used [24], taking into account age, gender, SBP > 160 mm HG, diabetes, cardiovascular disease (ischemic heart disease, heart failure, cardiomyopathy and peripheral arterial disease), stroke (chronic stroke) and chronic kidney disease status. Similar approaches have been used in previous clinical trials of self-monitoring in the control of hypertension $[15,16]$.

\section{Participant flow through the study}

An overview of the schedule of enrolment, interventions, and assessments in the ADAMPA study, according to the SPIRIT guidelines is shown in Fig. 1. Each aspect will be described in more detail throughout the present protocol.

Recruited hypertensive patients who meet the inclusion criteria and none of the exclusion criteria, who have been duly informed (by their GP) of the characteristics of the study, have signed the informed consent and been randomly assigned to the intervention or control group, will proceed as follows:

\section{Intervention group}

At their practice, the GPs will have established, with each patient in the intervention group, the BP target according to the European Guide for the management of Hypertension 2013 [19] and how to act according to their BP measurements (Fig. 2), including instructions for medication self-adjustment (if necessary). At the same time, the GP will inform them that they will be recalled to make an appointment with a member of the research team, who will provide them with additional information about their self-management of $\mathrm{BP}$ and for completing data corresponding to the baseline visit.

Subsequently, patients will be given -and trained in the use of- a validated home blood pressure monitor (Omron M3 model HEM-7131-E), as well as the "Intervention group booklet" containing: the patient's code, the reference arm on which BP is measured, the BP target, general information and basic recommendations for improving BP control, instructions to manage the home blood pressure monitor, how to act according to their BP (Fig. 2) and the "monthly registration sheets" for a six month period in order to register their blood pressure twice a day, once in the morning and once in the evening (for the first seven days of each month) and to register contacts related to their BP (by phone, regular or urgent consultation at the office or hospital consultation) during that follow-up period. 


\begin{tabular}{|c|c|c|c|c|c|c|}
\hline \multirow[b]{3}{*}{ TIMEPOINT } & \multicolumn{6}{|c|}{ STUDY PERIOD } \\
\hline & \multirow{2}{*}{$\begin{array}{c}\text { Enrolment } \\
-t_{1}\end{array}$} & \multirow{2}{*}{\begin{tabular}{|c|} 
Allocation \\
0
\end{tabular}} & \multicolumn{3}{|c|}{ Post-allocation } & \multirow{2}{*}{$\begin{array}{c}\begin{array}{c}\text { Follow } \\
\text { up }\end{array} \\
24 \text { mo } \\
\text { visit }\end{array}$} \\
\hline & & & $\begin{array}{l}\text { Base- } \\
\text { line } \\
\text { visit }\end{array}$ & $\begin{array}{l}6 \text { mo } \\
\text { visit }\end{array}$ & $\begin{array}{c}12 \\
\text { mo } \\
\text { visit }\end{array}$ & \\
\hline \multicolumn{7}{|l|}{ ENROLMENT: } \\
\hline \multirow[t]{3}{*}{ Eligibility screen } & $x$ & & & & & \\
\hline & $\mathrm{x}$ & & & & & \\
\hline & & $x$ & & & & \\
\hline \multicolumn{7}{|l|}{ INTERVENTIONS: } \\
\hline \multicolumn{7}{|l|}{$\begin{array}{l}\text { Self-monitoring blood } \\
\text { pressure and self-titration }\end{array}$} \\
\hline \multicolumn{7}{|l|}{ Control group (usual care) } \\
\hline \multicolumn{7}{|l|}{ ASSESSMENTS: } \\
\hline \multicolumn{7}{|l|}{$\begin{array}{r}\text { Sociodemographic } \\
\text { characteristics, co- } \\
\text { morbidities, year of onset of } \\
\text { hypertension, previous } \\
\text { SMBP }\end{array}$} \\
\hline \multirow{2}{*}{$\begin{array}{r}\text { Blood pressure (SBP and } \\
D B P \text { ) } \\
\text { Lifestyle (smoking, exercise, } \\
B M I)\end{array}$} & & & $x$ & $x$ & $x$ & $x$ \\
\hline & & & $x$ & $x$ & $x$ & \\
\hline \multirow{3}{*}{$\begin{array}{r}\text { Antihypertensive treatment } \\
\text { Number of concomitant } \\
\text { treatment }\end{array}$} & & & $x$ & $x$ & $\mathrm{x}$ & \\
\hline & & & $x$ & $x$ & $x$ & \\
\hline & & & $x$ & $x$ & $x$ & $x$ \\
\hline $\begin{array}{r}\text { Health services utilization } \\
\text { Health-related quality of life } \\
\text { (EQ5D) }\end{array}$ & & & $x$ & $x$ & $x$ & $x$ \\
\hline \multirow{2}{*}{$\begin{array}{r}\text { Clinical events } \\
\text { MAIN OUTCOME MEASURE: } \\
\text { Change in mean SBP } \\
\text { (mmHg) between baseline } \\
\text { and } 12 \mathrm{mo} \text { visit. }\end{array}$} & & & & $x$ & $x$ & $x$ \\
\hline & & & & & & \\
\hline \multicolumn{7}{|l|}{$\begin{array}{r}\text { SECONDARY OUTCOME } \\
\text { MEASURES: }\end{array}$} \\
\hline \multirow{2}{*}{ Change in mean SBP } & & & $\leftarrow$ & $\longrightarrow$ & & 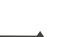 \\
\hline & & & & $\vec{\longrightarrow}$ & 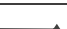 & \\
\hline \multirow{2}{*}{$\begin{array}{r}\text { Change in mean DBP } \\
\text { Percentage of patients with } \\
S B P<140 \mathrm{mmHg} \text { and } \\
D B P<90 \mathrm{mmHg}\end{array}$} & & & $\leftarrow$ & & & $\rightarrow$ \\
\hline & & & & $\mathrm{x}$ & $\mathrm{x}$ & $\mathrm{x}$ \\
\hline $\begin{array}{r}\text { Quality of life (as measured } \\
\text { by EuroQoL-5D) }\end{array}$ & & & & $\mathrm{x}$ & $\mathrm{x}$ & $\mathrm{x}$ \\
\hline $\begin{array}{r}\text { Adherence measured by } \\
\text { PDC }\end{array}$ & & & & $\mathrm{x}$ & $\mathrm{x}$ & \\
\hline \multirow[t]{2}{*}{ Persistence* $^{*}$} & & & & $x$ & $x$ & \\
\hline & & & & $x$ & $x$ & \\
\hline
\end{tabular}

BP: Blood pressure; SMBP: Self-monitoring blood pressure; SBP: Systolic Blood Pressure; DBP: Diastolic Blood Pressure; PDC: Proportion of Days Covered; * Defined as period of continuous use of the corresponding drug from the beginning of the follow-up until its discontinuation. ** Defined as the number of patients whose pharmacological treatment had not been modified divided by the number of patients not reaching the target values (SBP and/or DBP divided by the number of patients not reaching the target values (SBP and/or DBP
measurements taken at 6 and 12 months of follow-up), according to the recommendations of the European Society of Hypertension and European Society of Cardiology.

Fig. 1 (See legend on next page.) 
(See figure on previous page.)

Fig. 1 SPIRIT flow diagram: Schedule of enrolment, interventions, and assessments in the ADAMPA study. BP Blood pressure, SMBP Self-monitoring blood pressure, SBP Systolic Blood Pressure, DBP Diastolic Blood Pressure, PDC Proportion of Days Covered. * Defined as period of continuous use of the corresponding drug from the beginning of the follow-up until its discontinuation. ** Defined as the number of patients whose pharmacological treatment had not been modified, divided by the number of patients not reaching the target values (SBP and/or DBP measurements taken at 6 and 12 months of follow-up), according to the recommendations of the European Society of Hypertension and European Society of Cardiology

\section{Control group}

Patients will be informed by their GP that they will continue their usual care regarding their BP and that they will be recalled to make an appointment with a member of the research team, who will provide them with information and basic recommendations for improvement of $\mathrm{BP}$ control and for completing the data corresponding to the baseline visit. Subsequently, members of the research team will deliver the "Control group booklet" containing the patient's code, general information and basic recommendations for improving BP control, as well as the "monthly registration sheets" for a six month period in order to register contacts related to their BP (by phone, regular or urgent consultation at the general practice or hospital consultation) during the follow-up period.

Patients in the control and intervention groups will be informed that the research team will phone them four weeks after the baseline visit to clarify any doubts raised. If necessary, on-site visits will be arranged for further clarification.

Both groups will be contacted by phone at 3 months to clarify any doubts and at 6 months a follow-up visit will be established at the PCC, where the corresponding variables will be collected. The same will be done at 12 months. The follow-up variables will be collected up to a maximum of 6 weeks after the end of the follow-up period. An extension of the study will be performed with passive follow-up at 24 months, collecting a reduced set of outcome variables as secondary variables. Participants' flow through the trial is outlined in Fig. 3.

Patients have the right to leave the study at any time. In addition, the researcher may discontinue a patient from the study if deemed necessary for any reason including: non-eligibility (retrospective if not detected at the time of

\begin{tabular}{|c|c|c|c|}
\hline \multicolumn{4}{|c|}{ HOW TO ACT ACCORDING TO YOUR BLOOD PRESSURE MEASUREMENTS } \\
\hline \multicolumn{4}{|c|}{$\begin{array}{l}\text { Remember: } \\
\text { - Take your blood pressure twice a day, once in the morning and once in the evening, FOR the first seven days of each month. } \\
\text { - Whenever your BP is taken, you should take it twice, waiting for } 1-2 \text { minutes in between. } \\
\text { - In the case of a strange value, or a possible error in a measurement, repeat it to get } 2 \text { valid measurements. Once you have entered your } \\
\text { blood pressure values in your monthly notebook, act according to the following TABLE OF COLOURS OF ACTION, unless your doctor has } \\
\text { indicated otherwise. }\end{array}$} \\
\hline & $\begin{array}{l}\text { BLOOD PRESSURE VALUES } \\
\text { (in the morning or in the afternoon) }\end{array}$ & READING & ACTION \\
\hline VERY HIGH & $\begin{array}{l}\text { Your systolic blood pressure (TOP reading) is } \mathbf{1 8 0} \text { or } \\
\text { more } \\
\text { Your diastolic blood pressure (BOTTOM reading) is } \mathbf{1 0 0} \\
\text { or more }\end{array}$ & $\begin{array}{l}\text { Your blood pressure is } \\
\text { too high }\end{array}$ & $\begin{array}{l}\text { Contact your doctor or visit your health } \\
\text { care centre. }\end{array}$ \\
\hline HIGH & $\begin{array}{l}\text { If your systolic blood pressure (TOP reading) or your } \\
\text { diastolic blood pressure (BOTTOM reading) is above the } \\
\text { target values marked by your doctor. }\end{array}$ & $\begin{array}{l}\text { Your blood pressure is } \\
\quad \text { raised } \\
\text { If FouR or more readings a } \\
\text { week, once a month were } \\
\text { high, proceed with the } \\
\text { medication change } \\
\text { instructions. }\end{array}$ & $\begin{array}{l}1^{\text {st }} \text { step: Follow instructions provided by } \\
\text { your doctor. } \\
2^{\text {nd }} \text { step: make an appointment with your } \\
\text { doctor within } 3 \text { weeks after self- } \\
\text { adjustment. }\end{array}$ \\
\hline NORMAL & $\begin{array}{l}\text { Your blood pressure values are in the range of your BP } \\
\text { target. }\end{array}$ & $\begin{array}{l}\text { Your blood pressure is well } \\
\text { controlled }\end{array}$ & $\begin{array}{l}\text { Continue with your usual medication and } \\
\text { when you go to visit your doctor for any } \\
\text { reason, show your doctor your blood pressure } \\
\text { booklet measurements. }\end{array}$ \\
\hline Low & $\begin{array}{l}\text { Your systolic blood preassure (TOP reading) is } \mathbf{1 0 0} \text { or } \\
\text { less. }\end{array}$ & $\begin{array}{l}\text { Your blood pressure is } \\
\text { too low }\end{array}$ & $\begin{array}{l}\text { Contact your doctor or visit your health } \\
\text { care centre. }\end{array}$ \\
\hline \multicolumn{4}{|c|}{$\begin{array}{l}\text { RED: Contact your doctor or visit your health centre. } \\
\text { YELLOW: Proceed to self-adjustment at home with your doctor's instructions and schedule an appointment for } 3 \text { weeks after self-adjustment. }\end{array}$} \\
\hline \multicolumn{4}{|c|}{ IN CASE OF DOUBT, CONTACT YOUR GENERAL PRACTITIONER. } \\
\hline
\end{tabular}

Fig. 2 Instructions to patients: "HOW TO ACT ACCORDING TO YOUR BLOOD PRESSURE MEASUREMENTS" in the ADAMPA study. Adapted and modified from: The Colour Coding Chart. Supplementary webappendix in: McManus RJ, Mant J, Bray EP, et al. Telemonitoring and self-management in the control of hypertension (TASMINH2): a randomised controlledtrial. Lancet 2010; published online July 8. DOI:10.1016/S0140-6736(10)60964-6 


\section{Selection of participants}

Made by General Practitioners during practice visits (case-finding) until the target number of patients per GP is reached according to the previously established method.

- Hypertensive patients with $\mathrm{SBP}>145$ o $\mathrm{DBP}>90 \mathrm{~mm} \mathrm{Hg}$

- Age equal to or older than 40 years.

- Both genders

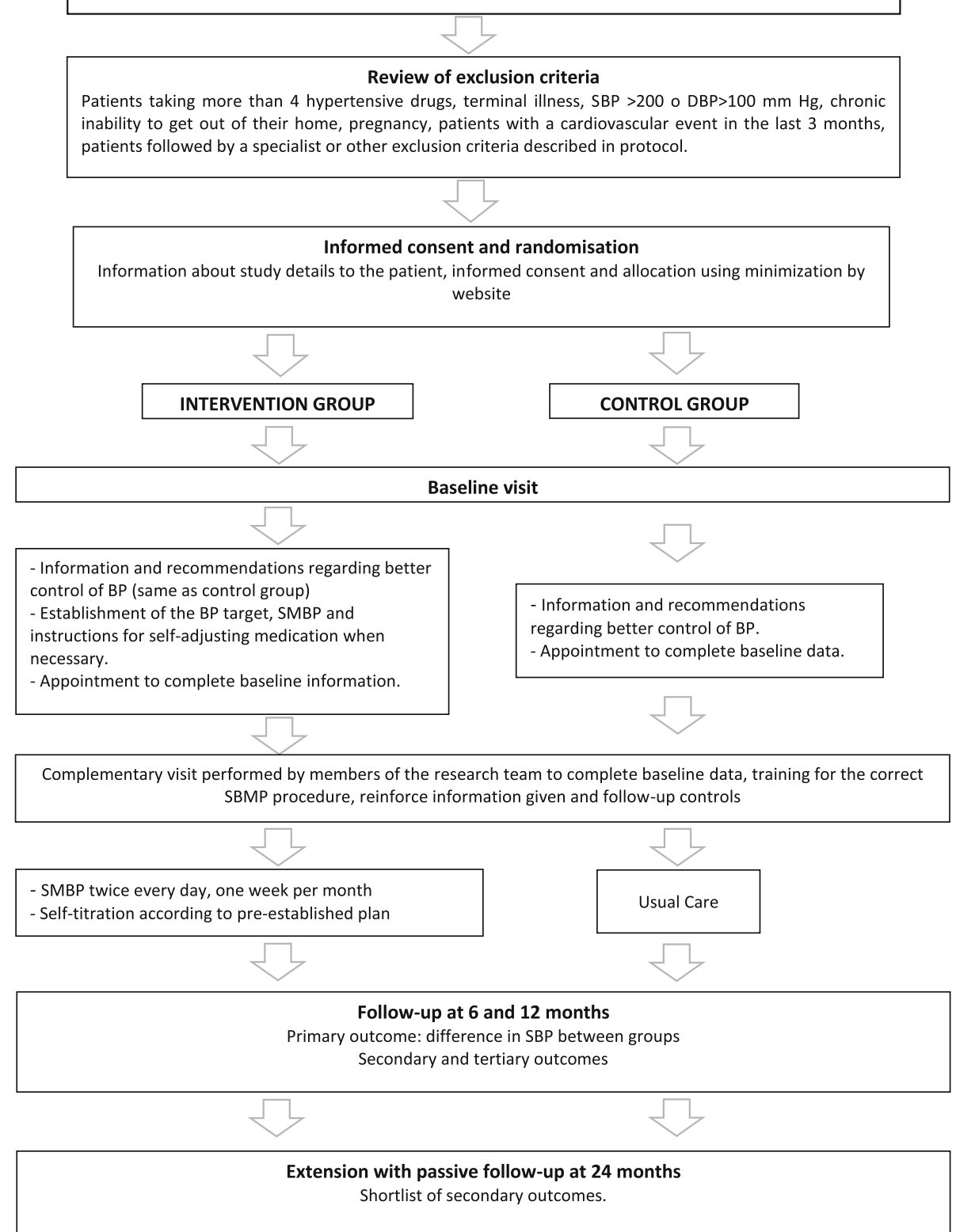

Fig. 3 Participants' flow through the study. GP General Practitioner, SBP Systolic Blood Pressure, DBP Diastolic Blood Pressure, BP Blood Pressure, SMBP Self-Management of Blood Pressure

inclusion, or prospective e.g. pregnancy during the follow up), an adverse event or disease progression involving incapacity to comply with trial procedures.

\section{Sample size consideration}

A sample size of 382 patients was estimated in order to have $90 \%$ power to detect a difference in SBP of $5 \mathrm{mmHg}$
(SD $15 \mathrm{mmHg}$ ) between the intervention and the control group with a contrast of two-tailed hypotheses and an alpha error of 0.05 . This figure represents a clinically relevant difference (which should represent a reduction of approximately 19\% in strokes) and is in line with the results observed in previous trials in this field (TASMINH2 and TASMINH-SR) $[15,16]$. These figures will 
be increased by $20 \%$ to compensate for possible drop-outs and follow-up loss, resulting in a total sample size of 458 participants.

\section{Intervention}

Intervention group

Blood pressure self-monitoring Patients will be trained to perform SMBP by the research team through a validated home blood pressure monitor (Omron model M3 HEM-7131-E). Patients will take their BP in the morning and in the afternoon, every day of the first week of each month. This will be done in the morning, before breakfast and before taking their medication (between $6 \mathrm{am}$. and $9 \mathrm{am}$.) and in the evening before dinner and before taking their medication (between $6 \mathrm{pm}$. and $9 \mathrm{pm}$.). These measurements will be recorded by the patients for the first seven days of each month on the "monthly registration sheets" located in the "Intervention group booklet". If patients want to monitor their BP during the remaining weeks of the month, it is recommended that they just do so one day a week. Patients are instructed to act according to a table that contains easy-to-follow colour coded action steps. This guideline prompts the patient to contact the GP or visit the health center when $\mathrm{BP}$ values are very high or very low. Four or more above target readings in a month will require a change in medication (See Fig. 2).

Target blood pressure Patients will be informed of their target BP, which will be established by their own GP and individualized for each patient based on the Guidelines for the management of arterial hypertension of the European Society of Hypertension (ESH) and European Society of Cardiology (ESC) [19]. Recommendations on target BP, according to cardiovascular risk and reflecting home as compared to office readings are shown in Table 1.
Self-titration In order to reach their target BP, each patient will be given a self-management plan to adjust medication if necessary depending on blood pressure measurements (See Fig. 2). The self-adjustment plan will consist of either an increase in the dose or additional medication. Therapeutic plan choice will be at the discretion of the GP, who will receive a copy of the Clinical Practice Guidelines of the European Society of Cardiology [19] to aid decision-making. If self-adjustment takes place, the participant will have an appointment with his/her GP within 3 weeks following self-adjustment, and a new tailored self-management plan will be provided.

\section{Control group}

Patients allocated to the control group will receive routine hypertension care with appointments and medication changes following the GP's criteria in the context of routine clinical practice.

In both, the intervention and control group, all relevant concomitant care within usual clinical practice will be at the discretion of the GP.

\section{Outcomes}

The primary outcome will be the change in mean SBP -mmHg- between baseline and 12 months.

Secondary outcomes will include:

1. Change in mean SBP at 6 and 24 months of follow-up.

2. Change in mean DBP at 6,12 , and 24 months of follow-up.

3. Percentage of patients with SBP $<140 \mathrm{mmHg}$ and $\mathrm{DBP}<90 \mathrm{mmHg}$ at 6,12 and 24 months of follow-up.

4. Quality of life (as measured by EuroQoL-5D) at 6, 12 and 24 months of follow-up.

5. Adherence measured by proportion of days covered (PDC) at 6 and 12 months of follow-up.

Table 1 Target blood pressure, according to cardiovascular risk conditions

\begin{tabular}{|c|c|c|c|c|c|}
\hline \multirow[t]{3}{*}{ Age } & \multirow[t]{3}{*}{ Clinical situation } & \multicolumn{4}{|c|}{ TARGET BLOOD PRESSURE } \\
\hline & & \multicolumn{2}{|l|}{ SBP } & \multicolumn{2}{|l|}{$\mathrm{DBP}$} \\
\hline & & SMBP & Office readings & SMBP & Office readings \\
\hline \multirow[t]{6}{*}{$<80$ years old } & Without increased cardiovascular risk & $\leq 135$ & $\leq 140$ & $<85$ & $<90$ \\
\hline & Diabetes & $<135$ & $<140$ & $<80$ & $<85$ \\
\hline & Cerebrovascular disease (previous stroke or TIA) & & & $<85$ & $<90$ \\
\hline & Coronary Heart Disease & & & & \\
\hline & Peripheral artery disease & & & & \\
\hline & Chronic kidney disease & & & & \\
\hline$\geq 80$ years old & & $<145$ & $<150$ & $<85$ & $<90$ \\
\hline
\end{tabular}

SBP Systolic blood pressure, DBP Diastolic blood pressure, SMBP Self measured blood pressure Modified from the 2013 ESH/ESC Guidelines for the management of arterial hypertension. The European Society of Hypertension (ESH) and European Society of Cardiology (ESC) 
6. Persistence, defined as period of continuous use of the corresponding drug from the beginning of the follow-up until its discontinuation at 6 and 12 months of follow-up.

7. Therapeutic inertia (TI), defined as the number of patients whose pharmacological treatment had not been modified, divided by the number of patients not reaching the target values (SBP and/or DBP measurements taken at 6 and 12 months of follow-up), according to the recommendations of the European Society of Hypertension and European Society of Cardiology [19].

Other outcome measures:

1. Changes in lifestyle (smoking, exercise, body weight) at 6,12 , and 24 months compared to these characteristics at baseline.

2. Clinical events: We will assess if any of the following adverse events are present during the follow-up: angina, myocardial infarction, stroke, hypotensive crisis and death.

3. Use of health services for hypertension at 6,12 and 24 months.

4. Incremental cost per quality-adjusted life year gained in the intervention group compared to the control group.

5. Views and experiences of patients and health professionals on the self-management (SMBP plus self-titration) of hypertension.

\section{Data collection}

Data will be collected at the different participant study sites. Details on type of data and timing of collection are shown in Fig. 1.

Data entry, coding, security, and storage, including any related processes to promote data quality (eg, double data entry, etc) and other aspects related to data management such as data monitoring of the ADAMPA study, will be performed by the SCReN platform (for more information on the Screen platform and its role regarding the ADAMPA study, see: https://www.scren.es/, and https://www.scren.es/proyectos.php).

\section{Statistical analysis}

Analysis will be on an intention-to-treat basis for complete cases. We will use mixed models (general linear modeling -GLM) to compare SBP at 12 months between the intervention and control groups. This analysis will be presented in both crude and adjusted forms for the different covariates of interest (baseline BP, gender, GP/PCC-random effect, diabetes, etc.). A sensitivity analysis will be performed to examine the potential effect of missing data, which will include substitution by multiple imputation, replacement of data lost by the most recent data or by the mean of the series. Additionally, analyses of the main outcome measure by subgroups of age, gender, comorbidity, level of chronicity, better control at baseline, etc. will be performed.

Differences in secondary outcome measures (DBP, percentage of patients controlled, PDC, persistence and TI) will be analyzed using methods similar to those used for analysis of the main outcome measure.

\section{Economic analysis}

The economic analysis will include a cost-consequence analysis, estimating both the costs (hospitalizations, outpatient visits, emergency visits and antihypertensive drugs) and the potential benefits (e.g. reduced incidence of stroke, myocardial infarction, etc.) in natural units. In addition, we will collect information on Health-Related Quality of Life (HRQOL) through the EQ-5d questionnaire, which will allow us to obtain utilities and therefore perform a cost-utility analysis with the estimated benefits in terms of Quality-Adjusted Life-Years (QALY).

A modeling will be performed to obtain longer-term predictions of the results observed in the trial. The results on which this modeling will be based will be survival, quality of life and costs associated with clinical events. A sensitivity analysis (deterministic and probabilistic) will be performed to analyze the robustness of the results. Key parameters will be modified to determine their impact on results. All analyses will be performed using STATA version 14.

\section{Quality sub-study}

Qualitative research techniques will seek to provide an in-depth understanding of the positive elements and areas of improvement related to self-titration and self-monitoring intervention. To this end, two meetings will be held, one with professionals (GPs and nurses) and one with patients, using the Nominal Group Technique (NGT). The NGT is a working methodology that establishes a framework for highly structured interaction that enables participation and equal consideration of the contributions of all members of the working group, and allows the identification of priorities, consensus and disagreement, solution generation and decision-making in an agile and objective manner [25].

\section{Discussion}

The ADAMPA trial is a clinical research project that aims to improve the control of BP through training the patient for self-management of their hypertension. Hypertension is a risk factor of high prevalence that, even today, presents an unacceptable percentage of uncontrolled patients, according to the recommendations of the guidelines of clinical practice for BP control. 
If the data from this trial show positive results, the study may contribute to a change of strategy in the treatment of hypertension, focusing on the patient as the main actor to achieve these objectives. Furthermore, this approach might contribute to the financial sustainability of the National Health Service.

\section{Abbreviations}

BP: Blood pressure; DBP: Diastolic blood pressure; GP: General practitioner; mm Hg: Millimeter of mercury; NGT: Nominal Group Technique; PC: Primary care; PCC: Primary care centers; PDC: Proportion of days covered;

SBP: Systolic blood pressure; SD: Standard deviation; SMBP: Self-measured blood pressure monitoring at home; TI: Therapeutic inertia

\section{Acknowledgements}

The members of the ADAMPA group (not listed individually in the author list of the tittle page) are as follows:

Joaquín, Abad-Carrasco; Maria V, Agudo-Escagüés; Marta, Alvarez-Martinez; Rosa M, Bartual-Penella; Rosa, Carrión-Villanueva; Encarnación, Checa-Sanz; Ana, Costa- Alcaraz; Isabel, Cristófol-López; Aurelio, Duque-Valencia; Rosario, González-Candelas; Ricardo, González-Espadas; Luis, González-Luján; Victoria, Gosalbes; Enrique, Guinot- Martínez; Emilio L, López-Torres; Silvia, MollaLLosa; Víctor, Moreno-Comins; Miriam, Moreno-Prat; María J, PuchadesCompany; Ángela, Ramos-García; Paloma, Ramos-Ruiz; Ester, Robles-Pastor; Pilar, Roca-Navarro; Rosa, Saiz-Rodriguez; Julia L, Salanova- Chilet; Ana, Tchang-Sanchez; Francisca, Torres; Ruth, Uribes-Fillol; Cristina, Valle-García; Macarena, Villar-Ruiz; Cristina, Vivas-Miquel.

\section{Funding}

The trial is funded by the Carlos III Health Institute (Grant PI16/02130 and the SCReN-Spanish Clinical Research Network, PT13/0002/0031), the Spanish Ministry of Economy and Competitiveness. Co-financed by the European Regional Development Fund. No agreement limits the access to data for investigators. The funding agencies did not have any role in the design of the study, collection, analysis, interpretation of data, and in writing the manuscript

\section{Trial sponsor}

Instituto de investigación Sanitaria INCLIVA.

Avda. Menéndez Pelayo, 4 Acc.

46,010 Valencia.

Contact person: Marta Peiró Signes. Scientific Subdirector INCLIVA.

\section{Authors' contributions}

JSG conceived the study. JSG, GSG, AGS and SP carried out the design of the study. JSG, CLRB and GSG drafted the manuscript. IMM, PMI, LML, MBG, IBF, MCDS, CBM, EAH, MEV, ML, LBP, and ADAMPA group are involved in the acquisition of data and contributed to the design of the study. All authors (JSG, GSG, AGS, SP, CLRB, IMM, PMI, LMI, MBG, IBF, MCDS, CBM, EAH, MEV, $M L, M G L, L B P$, and ADAMPA group) contributed to the critical revision of the manuscript for important intellectual content, and all authors read and approved the final manuscript.

\section{Ethics approval and consent to participate}

This trial obtained full approval from the University Clinical Hospital Ethics Committee (CEIC-HCUV) and from the Spanish Agency for Medication and Health Products (AEMPS). GPs will give the information sheet and the informed consent form to each patient, responding to all queries and concerns. All patients voluntarily accepting to participate will provide the corresponding informed consent signed. Important protocol modifications (eg, changes to eligibility criteria, outcomes, analyses) will be communicated to the different involved parties (eg, investigators, REC/RBBs, trial participants, trial registries, journals, regulators), depending on the relevance.

\section{Consent for publication}

Not applicable.

\section{Competing interests}

The authors declare that they have no competing interests.

\section{Publisher's Note}

Springer Nature remains neutral with regard to jurisdictional claims in published maps and institutional affiliations.

\section{Author details}

${ }^{1}$ Centro de Salud de Nazaret, Departamento de Salud de Valencia Clínic-La Malvarrosa, Valencia, Spain. ${ }^{2} H e a l t h$ Services Research Unit, FISABIO, Valencia, Spain. ${ }^{3}$ Spanish Network of Chronic Care and Health Services Research (REDISSEC), Valencia, Spain. ${ }^{4}$ Health Research Institute (INCLIVA), Valencia, Spain.

Received: 26 March 2018 Accepted: 3 September 2018 Published online: 24 September 2018

\section{References}

1. Murphy T, Naghavi M, Neal B, Lim SS, Vos T, Flaxman AD, et al. A comparative risk assessment of burden of disease and injury attributable to 67 risk factors and risk factor clusters in 21 regions, 1990-2010: a systematic analysis for the global burden of disease study 2010. Lancet. 2012;380(9859): 2224-60.

2. Catalá-López F, Sanfélix-Gimeno G, García-Torres C, Ridao M, Peiró S. Control of arterial hypertension in Spain: a systematic review and meta-analysis of 76 epidemiological studies on 341632 participants. J Hypertens. 2012;30(1): $168-76$.

3. Catalá-López F, Ridao M, Sanfélix-Gimeno G, Peiró S. Trends of uncontrolled blood pressure in Spain: an updated meta-regression analysis. J Hypertens. 2013;31(3):630-1.

4. Joffres M, Falaschetti E, Gillespie C, Robitaille C, Loustalot F, Poulter N, et al. Hypertension prevalence, awareness, treatment and control in national surveys from England, the USA and Canada, and correlation with stroke and ischemic heart disease mortality: a cross-sectional study. BMJ Open. 2013; 3(8):e003423.

5. Redon J, Olsen MH, Cooper RS, Zurriaga O, Martinez-Beneito MA, Laurent S, et al. Stroke mortality and trends from 1990 to 2006 in 39 countries from Europe and Central Asia: implications for control of high blood pressure. Eur Heart J. 2011:32(11):1424-31.

6. Zhao Y, Yan H, Marshall RJ, Dang S, Yang R, Li Q, et al. Trends in population blood pressure and prevalence, awareness, treatment, and control of hypertension among middle-aged and older adults in a rural area of Northwest China from 1982 to 2010. PLoSOne. 2013;8(4):e61779.

7. Guo F, He D, Zhang W, Walton RG. Trends in prevalence, awareness, management, and control of HypertensionAmong United States adults, 1999 to 2010. J Am Coll Cardiol. 2012;60(7):599-606.

8. Banegas JR, Graciani A, de la Cruz-Troca JJ, León-Muñoz LM, GuallarCastillón P, Coca A, et al. Achievement of cardiometabolic goals in aware hypertensive patients in Spain: a nationwide population-based study. Hypertension 2012;60(4):898-905.

9. Borghi C, Tubach F, De Backer G, Dallongeville J, Guallar E, Medina J, et al. Lack of control of hypertension in primary cardiovascular disease prevention in Europe: results from the EURIKA study. Int J Cardiol. 2016;218:83-8.

10. Cifu AS, Davis AM. Prevention, detection, evaluation, and Management of High Blood Pressure in adults. JAMA. 2017;318(21):2132-4.

11. McManus RJ, Wood S, Bray EP, Glasziou P, Hayen A, Heneghan C, et al. Selfmonitoring in hypertension: a web-based survey of primary care physicians. J Hum Hypertens. 2014;28(2):123-7.

12. Logan AG, Dunai A, Mclsaac WJ, Irvine MJ, Tisler A. Attitudes of primary care physicians and their patients about home blood pressure monitoring in Ontario. J Hypertens. 2008;26(3):446-52.

13. Glynn LG, Murphy AW, Smith SM, Schroeder K, Fahey T. Interventions used to improve control of blood pressure in patients with hypertension. Cochrane Database Syst Rev. 2010;3. CD005182.

14. Uhlig K, Patel K, Ip S, Kitsios GD, Balk EM. Self-measured blood pressure monitoring in the management of hypertension: a systematic review and meta-analysis. Ann Intern Med. 2013;159(3):185-94.

15. McManus RJ, Mant J, Bray EP, Holder R, Jones MI, Greenfield S, et al. Telemonitoring and self-management in the control of hypertension (TASMINH2): a randomized controlled trial. Lancet. 2010;376(9736):163-72.

16. McManus RJ, Mant J, Haque MS, Bray EP, Bryan S, Greenfield SM, et al. Effect of self-monitoring and medication self-titration on systolic blood pressure in hypertensive patients at high risk of cardiovascular disease: the TASMIN-SR randomized clinical trial. JAMA. 2014;312(8):799-808. 
17. Margolius D, Bodenheimer T, Bennett H, Wong J, Ngo V, Padilla G, et al. Health coaching to improve hypertension treatment in a low-income, minority population. Ann Fam Med. 2012;10:199-205.

18. James PA, Oparil S, Carter BL, Cushman WC, Dennison-Himmelfarb C, Handler J, et al. 2014 evidence-based guideline for the management of high blood pressure in adults: report from the panel members appointed to the eighth joint National Committee (JNC 8). JAMA. 2014;311(5):507.

19. Mancia G, Fagard R, Narkiewicz K, Redón J, Zanchetti BM, et al. 2013 ESH/ ESC guidelines for the management of arterial hypertension. The Task Force for the management of arterial hypertension of the European Society of Hypertension (ESH) and of the European Society of Cardiology (ESC) J Hypertension. 2013;31:1281-357.

20. Navar-Boggan AM, Pencina MJ, Williams K, Sniderman AD, Peterson ED. Proportion of US adults potentially affected by the 2014 hypertension guideline. JAMA. 2014;311(14):1424-9.

21. The ACCORD Study Group. Effects of intensive blood-pressure control in type 2 diabetes mellitus. N Engl J Med. 2010;362:1575-85.

22. The SPRINT Research Group. A randomized trial of intensive versus standard blood-pressure control. N Engl J Med. 2015;373:2103-16.

23. Lonn EM, Bosch J, López-Jaramillo P, Zhu J, Liu L, Pais P, et al. (HOPE-3 investigators). Blood-pressure lowering in intermediate-risk persons without cardiovascular disease. N Engl J Med. 2016:374(21):2009-20.

24. Scott NW, McPherson GC, Ramsay CR, Campbell MK. The method of minimization for allocation to clinical trials. a review Control Clin Trials 2002 Dec;23(6):662-74. Review. PubMed PMID: 12505244.

25. Delbecq A, Van De Ven A. A group process model for problem identification and program planning. J Appl Behav Sci. 1971;7(4):466-92.

Ready to submit your research? Choose BMC and benefit from:

- fast, convenient online submission

- thorough peer review by experienced researchers in your field

- rapid publication on acceptance

- support for research data, including large and complex data types

- gold Open Access which fosters wider collaboration and increased citations

- maximum visibility for your research: over $100 \mathrm{M}$ website views per year

At $\mathrm{BMC}$, research is always in progress.

Learn more biomedcentral.com/submissions 